OnLine Journal of Biological Sciences 9 (2): 40-51, 2009

ISSN 1608-4217

(C) 2009 Science Publications

\title{
Enhanced Survival and Nodule Occupancy of Pigeon pea Nodulating Rhizobium sp. ST1 expressing fegA Gene of Bradyrhizobium japonicum $61 \mathrm{A152}$
}

\author{
${ }^{2}$ Falguni R. Joshi, ${ }^{2}$ Dhwani K. Desai, ${ }^{1}$ G. Archana and ${ }^{1}$ Anjana J. Desai \\ ${ }^{1}$ Department of Microbiology and Biotechnology Centre, Faculty of Science, \\ Maharaja Sayajirao University of Baroda, Vadodara, Gujarat, India \\ ${ }^{2}$ Centre for Computational Biology and Bioinformatics, School of Information Technology, \\ Jawaharlal Nehru University, New Delhi, India
}

\begin{abstract}
Problem statement: Rhizobial isolates belonging to genera (Rhizobium sp. and Mesorhizobium sp.) in our laboratory produced only catecholate type of siderophores. Although FhuA and FegA (ferrichrome receptors) homologs were found to be present in the sequenced genomes of few rhizobia (e.g., 1 in R. etli and 2 in Mesorhizobium sp. BNC1), laboratory isolates of the corresponding genera failed to utilize ferrichrome, a siderophore which is present in nanomolar concentrations in the soil. This inability was considered as a negative fitness factor with respect to rhizospheric colonization by these rhizobia. Approach: The $2.4 \mathrm{~kb} \mathrm{fegA}$ gene (encoding ferrichrome receptor) was amplified along with its native promoter from Bradyrhizobium japonicum 61A152 and cloned in a broad host range plasmid vector pUCPM18. The plasmid construct $\mathrm{pFJ}$ was transferred by conjugation into Rhizobium sp. ST1 to give transconjugant ST1pFJ12. The consequence of FegA expression on the transconjugant was tested under lab and soil conditions, using physiological experiments. Results: Ability of the transconjugant ST1pFJ12 to utilize ferrichrome and expression of a $79 \mathrm{kD}$ protein band on the outer membrane of the transconjugant confirmed FegA expression. Transconjugant ST1pFJ12 exhibited increased growth rate as compared to the parent strain ST1, in minimal media containing ferrichrome as the sole iron source, confirming the positive effect of FegA expression. Inoculation of pigeon pea seedlings with transconjugant ST1pFJ12 led to a marked increase in plant growth parameters as compared to plants inoculated with the parent strain ST1, the effect being more pronounced when Ustilago maydis, a ferrichrome producer was co-inoculated in the systems. Nodule occupancy on pigeon pea plant when inoculated with the transconjugant ST1pFJ12 alone was 57\% which increased to $66 \%$ when co-inoculated with $U$. maydis as compared with 37 and $30 \%$ respectively, seen with parental strain ST1 inoculation. Conclusion: The clear increase in nodule occupancy and higher rhizospheric colonization by the $f e g A$ transconjugants, presented in this study together with the previous research reported from our laboratory, led us to conclude that ferrichrome utilization ability played an important role in the rhizospheric colonization of the bioinoculant strains. Testing the ability to utilize hydroxamate siderophores therefore, holds prime importance in selecting an efficient biofertilizer strain.
\end{abstract}

Key words: Ferrichrome, rhizobia, rhizospheric colonization, cross-utilization, nodule occupancy

\section{INTRODUCTION}

Although iron is the fourth most abundant element on the Earth, it is mainly present in its oxidized state, having a solubility of $10^{-18} \mathrm{M}$ at biological $\mathrm{pH}^{[1]}$, which is much less than what is required by most soil microorganisms. The solubility further decreases 1000 fold with increase in $\mathrm{pH}$ by 1 unit, due to its tendency to form iron-hydroxides polymers in aqueous environments ${ }^{[1]}$. Microorganisms, including rhizobia, employ various mechanisms to acquire this essential nutrient, which includes production of iron chelating molecules known as siderophores, which bind $\mathrm{Fe}^{+3}$ with a very high affinity and form ferrisiderophore complexes. These ferrisiderophore complexes are taken inside the cells, aided by multi-component iron uptake systems, comprising of a high-affinity outer membrane receptor, which is specific to the ferrisiderophore ligand, in association with broadly specific periplasmic binding proteins and inner membrane ATPases.

Corresponding Author: Anjana J. Desai, Department of Microbiology and Biotechnology Centre, Faculty of Science,

The Maharaja Sayajirao University of Baroda, Vadodara 390 002, Gujarat, India Tel: 91-265-2794396 
Rhizobia are amended into agricultural soils, but despite their efficient nitrogen fixing potential, most of the times they fail to increase plant yields, which is attributed to their inefficiency to successfully colonize the rhizosphere, iron availability being one of the limiting factors for rhizospheric colonization ${ }^{[6,7]}$.

Rhizobia are known to produce a wide variety of siderophores; Rhizobium meliloti DM4 and Sinorhizobium meliloti produce rhizobactin ${ }^{[8-10]}$, Rhizobium leguminosarum bv. viciae MNF7101 and WSM710 produce vicibactin ${ }^{[11]}$, catecholate siderophores viz., salicylic acid and dihydroxybenzoic acid are produced by $R$. ciceri isolates from chick pea nodules ${ }^{[12]}$ whereas uncharacterized catecholates are produced by rhizobia from the cowpea group ${ }^{[13,14]}$. In alkaline soils of the mid-western United States B. japonicum serotype 135, a siderophore producer, dominates over serotype 123 , a non-siderophore producer ${ }^{[15]}$. In addition to that, $54 \%$ of $S$. meliloti strains isolated from alfalfa nodules from a high $\mathrm{pH}$ soil were siderophore producers, whereas those from a soil of lower $\mathrm{pH}$, the proportion of siderophore-producing strains fell to $18 \%{ }^{[16]}$. The above facts underline the importance of iron acquisition systems in rhizobial populations.

Fluorescent pseudomonads are most studied Plant Growth Promoting Rhizobacteria (PGPR) and one of the traits contributing to their PGPR status is not only their ability to produce large number of siderophores but also their ability to utilize equal numbers of heterologous siderophores (siderophores produced by other organisms) via various TonB dependent siderophore receptors. Studies report 32 putative siderophore receptors in P. aeruginosa ${ }^{[17,18]}, 29$ in P. putida, 27 in P. fluorescens and 23 in $P$. syringae ${ }^{[19]}$. Amongst rhizobia B. japonicum $61 \mathrm{~A} 152$ is reported to be a successful bioinoculant strain for soybean crop ${ }^{[22]}$. Bradyrhizobium japonicum $61 \mathrm{~A} 152$ produces citrate as the only siderophore ${ }^{[20]}$, but also can internalize iron complexed with ferrichrome and rhodotorulic acid, the hydroxamate siderophores produced by many soil fungi ${ }^{[2]}$. This is attributed to the presence of $f e g A$ gene, encoding ferrichrome receptor, in this organism. The concentration of hydroxamate type siderophore in soil is reported to be as high as $10 \mu \mathrm{M}^{[23]}$, ferrichrome, constituting the major fraction amongst these ${ }^{[24]}$.

Most of the rhizobial isolates and other nodule bacteria tested in our laboratory produced mainly catecholate type of siderophores and failed to utilize iron complexed with ferrichrome, a hydroxamate siderophore known to be present in abundance in the soil ${ }^{[26-29]}$. Many studies indicate that efficient utilization of hydroxamate siderophores by rhizobia is a positive fitness factor with respect to its soil survival ${ }^{[22,45]}$. The objective of the present study therefore was to impart upon these rhizobia ferrichrome utilization ability so as to increase their competitive survival in rhizosphere.

Recent study from our laboratory has shown that the expression of Bradyrhizobium japonicum 61A152 $f e g A$ gene in peanut rhizobia, not only supports the growth of the $f e g A$ transformants under iron limited laboratory conditions, but also increases its survivability under natural soil conditions, which led to higher nodulation on peanut plant ${ }^{[29]}$. Similar study regarding the expression of $B$. japonicum fegA in pigeon pea rhizobia, Rhizobium sp. ST1, presented in this investigation would, not only substantiate the earlier studies, but also would allow us to generalise the previous observation that hydroxamate siderophore utilization ability confers upon a strain competitive survival and therefore can be considered as an important criteria while selecting an efficient bioinoculant strain. Studies have also been extended towards carrying out an in silico investigation of the occurrence of ferrichrome receptor genes in sequenced genomes of various rhizobia to substantiate our findings.

\section{MATERIALS AND METHODS}

Bacterial strains, plasmids and growth conditions: The bacterial strains and plasmids used in this study are as in Table 1. Rhizobium sp. ST1 (16SrRNA gene sequence Genbank Accession number DQ632608), a nitrogen fixing nodule symbiont of pigeon pea (Cajanus cajan) is a lab isolate; S. meliloti IC3169 (C. cajan); Mesorhizobium sp. GN25 (Arachis hypogea, 16SrRNA gene sequence Genbank Accession number DQ862066) ${ }^{[31]}$ and Bradyrhizobium sp. NC92 (A. hypogea) were procured from Indian Agricultural Research Institute (IARI), New Delhi, India; B. japonicum 61A152 was a kind gift from Guerinot, M.L., Dartmouth college, Hanover, USA ${ }^{[32]}$. All the above strains were maintained routinely on YEM medium ( $1 \%$ mannitol, $0.1 \%$ yeast extract, $0.02 \%$ $\mathrm{MgSO}_{4} .7 \mathrm{H}_{2} \mathrm{O}, 0.01 \% \mathrm{NaCl}, 0.05 \% \mathrm{~K}_{2} \mathrm{HPO}_{4}$ ). Ashby's Mannitol (AM) broth (1\% mannitol, $0.2 \%$ sodium glutamate, $0.02 \% \mathrm{MgSO}_{4} .7 \mathrm{H}_{2} \mathrm{O}, 0.01 \% \mathrm{NaCl}, 0.05 \%$ $\mathrm{K}_{2} \mathrm{HPO}_{4}$ ) deferrated using $0.25 \%$ 8-hydroxyquinoline in chloroform was used as iron limited media for all the rhizobial strains and ST1pFJ12, fegA transconjugant of Rhizobium sp. ST1. Iron supplemented medium was prepared by adding $100 \mu \mathrm{M} \mathrm{FeCl} 3$ to the above medium. The medium as described by Winkelmann was used for siderophore production by $U$. maydis and Rhodotorula mucilaginosa MTCC $850{ }^{[31]}$ P. fluorescens ATCC13525, P. aeruginosa MTCC2453 and P. putida KT2440 were from our laboratory collection; 
Table 1: Bacterial strains and plasmids used in this study

\begin{tabular}{|c|c|c|}
\hline Bacterial/fungal strains or plasmids & Relevant characteristics & Source or reference \\
\hline \multicolumn{3}{|l|}{ Rhizobial strains: } \\
\hline Rhizobium sp. ST1 & $\begin{array}{l}\text { Nitrogen fixing pigeon pea (Cajanus cajan) nodule symbiont, 16SrRNA } \\
\text { gene sequence accession number DQ632608, Rifampicin }{ }^{\mathrm{R}}\end{array}$ & Laboratory collection \\
\hline Mesorhizobium sp. GN25 & $\begin{array}{l}\text { Peanut (Arachis hypogea) nodule symbiont, 16SrRNA gene sequence } \\
\text { accession number DQ862066 }\end{array}$ & IARI, New Delhi, India \\
\hline Sinorhizobium sp. IC3169 & Sinorhizobium sp., pigeon pea biofertilizer strain & IARI, New Delhi, India \\
\hline Bradyrhizobium sp. NC92 & Bradyrhizobium sp. peanut biofertilizer strain & IARI, New Delhi, India. \\
\hline Bradyrhizobium japonicum 61A152 & Nitrogen-fixing Glycine max (soybean) symbiont & \\
\hline ST1pFJ12 & ST1 carrying pFJ & Present study \\
\hline \multicolumn{3}{|l|}{ Escherichia coli: } \\
\hline DH5 $\alpha$ & $\begin{array}{l}\text { hsdR17 endA1 thi-1 gyrA96 relA1 supE44 } \triangle l a c \\
\text { U169 ( }(\text { 80dlacZAM15) }\end{array}$ & [38] \\
\hline S17.1 & $\begin{array}{l}h s d R \text { pro recA containing RP-4-2-Tc:: } \mathrm{Mu} \\
\text { integration into chromosome }\end{array}$ & [39] \\
\hline S17.1pFJ & E. coli S17.1 carrying pFJ & Present study \\
\hline MB97pFJ & MB97 $\Delta f h u A$ carrying $\mathrm{pFJ}$ & This study \\
\hline \multicolumn{3}{|l|}{ Pseudomonas strains: } \\
\hline P. fluorescens ATCC 13525 & Used as a source of heterologous siderophores & Laboratory collection \\
\hline P. aeruginosa $\mathrm{MTCC} 2453$ & Used as a source of heterologous siderophores & Laboratory collection \\
\hline P. putida KT2440 & Used as a source of heterologous siderophores & Laboratory collection \\
\hline \multicolumn{3}{|l|}{ Plasmids } \\
\hline pTZ57R-T & Used for cloning the $f e g A$ amplicon & [MBI Fermentas] \\
\hline PTZfegA & fegA amplicon cloned in pTZ57R-T & Present study \\
\hline pUCPM18-Gm & $\begin{array}{l}\text { Escherichia-Pseudomonas shuttle vector pUCP19, } \\
\text { containing the mob fragment }\end{array}$ & \\
\hline $\mathrm{pFJ}$ & pUCPM18-Gm with $f e g A$ cloned in & $\begin{array}{l}\text { Present study } \\
\text { XbaI-BamHI site }\end{array}$ \\
\hline
\end{tabular}

positive for siderophore production and their supernatants at the end of $48 \mathrm{~h}$ of growth under iron limiting condition was used as sources of siderophores.

Whenever required, the following concentration of antibiotics was supplemented in the media-for rhizobia: Ampicillin (Amp $150 \mu \mathrm{g} \mathrm{mL}^{-1}$ ); rifampicin (Rif $50 \mu \mathrm{g} \mathrm{mL}^{-1}$ ) and gentamycin $\left(\mathrm{Gm} 80 \mu \mathrm{g} \mathrm{mL}^{-1}\right.$ ) and for E. coli: Ampicillin (Amp $100 \mu \mathrm{g} \mathrm{mL}^{-1}$ ); gentamycin $\left(\mathrm{Gm} 40 \mu \mathrm{g} \mathrm{mL}^{-1}\right)$.

Siderophore production and cross-utilization bioassay: Siderophore production was induced in all the rhizobial strains by growing them in deferrated AMB for $48 \mathrm{~h}$ and the supernatants were tested for the presence of catecholate ${ }^{[32]}$ and hydroxamate ${ }^{[33]}$ siderophores, using 2,3-dihydroxybenzoic acid and hydroxylamine hydrochloride respectively, as standards. Siderophore cross-utilization bioassay was performed exactly as described by ${ }^{[29]}$.

Sequence analysis: Sequences from various organisms annotated as FhuA, FhuB, FhuC and FhuD respectively, were collected from the SWISSPROT/TrEMBL ${ }^{[34]}$ database. The sequences for each group were aligned using MUSCLE ${ }^{[35]}$. The HMM-build_mw program was used to construct profile-HMMs from the alignments and the HMM-search program (E-value cut-off of 0.1) was used to scan the protein sequences in the genomes of Rhizobium etli CFN42, Sinorhizobium meliloti 1021,
Mesorhizobium loti MAFF303099, Mesorhizobium sp. BNC1, Rhizobium leguminosarum bv viciae 3841 and B. japonicum USDA110 along with their plasmid sequences. As the number of sequences of FegA and FegB were insufficient to construct HMMs, BLAST searches against the genomes were used to identify homologs ${ }^{[36]}$ (E-value cut-off of 0.1).

Primer designing and PCR amplification of fegA and construction of plasmid $\mathbf{p F J}$ : The $f e g A$ amplicon was cloned in pGEM-T vector (MBI Fermentas) and subcloned subsequently in pUCPM18-Gm ${ }^{[37]}$ exactly as described in ${ }^{[29]}$.

DNA manipulations: Genomic DNA isolation was done as described in ${ }^{[38]}$. Plasmid DNA isolations (from E. coli and rhizobia), DNA ligations, transformation of $E$. coli with plasmid DNA were also performed using standard procedures ${ }^{[38]}$. Restriction endonuclease digestions were performed according to manufacturer's instructions (MBI Fermentas). pFJ was transferred from S17.1 containing pFJ to Rhizobium sp. ST1 by patch mating method as described by ${ }^{[39]}$.

Growth assays: Initial inoculum was prepared by growing the cultures in YEM medium and 1\% of $1.0 \mathrm{OD}$ culture was inoculated in iron limited media. Pure ferrichrome was exogenously added to a final 
concentration of $15 \mu \mathrm{M}$. Growth was measured as optical density at $600 \mathrm{~nm}$ every $6 \mathrm{~h}$ post inoculation.

SDS-PAGE analysis of Outer Membrane Proteins (OMPs): OMP extraction was done by the method of ${ }^{40]}$ with minor modifications as described in $^{[29]}$ for rhizobial cultures.

Plant studies: Agricultural soil was collected from Anand agricultural university, Model farm, Vadodara, Gujarat. Analysis of soil parameters was done at Gujarat State Fertilizer Corporation, Vadodara, Gujarat, India. The soil was alkaline $(\mathrm{pH} 7.8$, with electrical conductivity of $0.27 \mathrm{mmho} \mathrm{cm}^{-1}$ and organic C content of $0.57 \mathrm{~g} \mathrm{~kg}^{-1}$. The total iron concentration was $14.66 \mathrm{ppm}$, as estimated by atomic absorption spectroscopy. The total siderophore concentration was estimated to be $3.8 \mu \mathrm{M}$ using CAS solution ${ }^{[41,42]}$. Pigeon pea [BDN-2, Gujarat, India] seeds were surface sterilized, coated with ST1 and ST1pFJ12 and the pot experiments were set up in the same way as done in ${ }^{[29]}$, but only under natural (un-autoclaved) soil conditions. The pots were incubated under natural day-light conditions and at the end of 30 days were checked for various parameters like shoot fresh weight, root fresh weight, chlorophyll content of the leaves and nodule density. Plants without inoculation of either rhizobial and/or fungal cultures were used as controls. The count of the parent strain ST1 (RifR) and transconjugant (RifR, AmpR, GmR) colonizing the rhizosphere was determined by plating appropriate dilutions of rhizospheric soil suspension $\{1: 1$ soil $(\mathrm{g})$ : Sterile saline $(\mathrm{mL})\}$ on YEM plates containing the appropriate antibiotics. Nodule occupancy by inoculated organisms was determined exactly as in ${ }^{[29]}$.

Statistical analysis: The statistical analysis of the results obtained was done by one way ANOVA (analysis of variance) using the web-trial version of SigmaStat 3.5. Null hypothesis was set on mean difference equal to 0 and Alpha at 0.05 . The difference between all the comparisons made is significant at $95 \%$ confidence interval.

\section{RESULTS}

Pseudomonads are known for their rhizospheric stability and one of the factors contributing to this is the presence of diverse iron uptake systems. About 32 putative siderophore receptors in $P$. aeruginosa ${ }^{[17,18]}$, 29 in $P$. putida, 27 in $P$. fluorescens and 23 in $P$. syringae ${ }^{[19]}$ are reported. Analysis of protein sequences of complete genomes of Pseudomonas species using HMMER profiles revealed the presence of 45 TonB dependent siderophore receptors in the genome of $P$. fluorescens, 31 in $P$. putida and 36 in $P$. aeruginosa. In contrast, a complete genome wide search in a few members of rhizobiales revealed a visible scarcity of TonB dependent siderophore receptors; 3 were present in R. etli, 3 in Mesorhizobium sp. BNC1, 2 in Mesorhizobium loti, 2 in Sinorhizobium meliloti and 8 in Bradyrhizobium japonicum (Table 2) Relatively high number of TonB dependent receptors present in Bradyrhizobium sp. amongst rhizobiales could be attributed for their high rhizospheric competence and hence their reported success as commercial biofertilizers for soybean crops ${ }^{[22]}$. This led us to conceptualize that increasing the repertoire of outer membrane siderophore receptors could make our rhizobial isolates more efficient with respect to iron acquisition and hence colonizing the rhizosphere. Laboratory rhizobial isolates Rhizobium sp. ST1, Mesorhizobium sp. GN25, Sinorhizobium sp. IC3169 were found to produce only catecholate type of siderophores (Table 3) and failed to utilize hydroxamate siderophores, mainly ferrichrome and rhodotorulic acid (Table 3). Since rhizobia generally lack the ability to produce and utilize hydroxamates ${ }^{[26-29]}$, it is hypothesized that imparting upon them the ability to utilize hydroxamate siderophores would lead to increase in their rhizosperic competence.

Table 2: In silico identification of TonB dependent siderophore receptors in complete genomes of pseudomonads and rhizobia

\begin{tabular}{lc}
\hline Organism whose sequence was taken & $\begin{array}{l}\text { No. of TonB dependent } \\
\text { receptors detected }\end{array}$ \\
\hline P. aeruginosa & 36 \\
P. fluorescens & 45 \\
P. putida & 31 \\
Mesorhizobium loti & 1 \\
Mesorhizobium sp. BNC1 & 3 \\
Rhizobium etli CFN42 & 3 \\
Sinorhizobium meliloti & 2 \\
R. leguminosarum & 3 \\
Bradyrhizobium japonicum USDA110 & 8 \\
\hline
\end{tabular}

Table 3: Siderophore production and cross-utilization by the rhizobial isolates

\begin{tabular}{llllllllll}
\hline & $\begin{array}{l}\text { Catecholate } \\
\text { siderophore } \\
\text { production }\end{array}$ & \multicolumn{1}{l}{ Siderophores tested for cross-utilization } & \\
Isolate & $\left(\mu \mathrm{g} \mathrm{mL}^{-1}\right)$ & FC & RA & Desf & PF & PA & PP & Fe-cit \\
\hline 61A152 & - & & + & + & + & - & - & + & + \\
ST1 & 15.32 & - & - & - & - & - & + & + \\
IC3169 & 2.21 & - & - & - & - & - & + & + \\
GN25 & 2.93 & - & - & + & - & - & + & + \\
NC92 & 3.02 & + & + & + & - & - & - & + \\
\hline
\end{tabular}

(+): indicates growth and (-): indicates no growth around the siderophore containing wells; FC: ferrichrome, RA: Rhodotorulic acid, Desf: desferal, PF: Pseudomonas fluorescens culture supernatant, PA: P. aeruginosa culture supernatant, PP: P. putida culture supernatant 
Table 4: Identification of ferri-ferrichrome uptake machinery (FegA, FhuA, FhuB, FhuC and FhuD) homologs from sequenced genomes of rhizobia

\begin{tabular}{lccrrrr}
\hline & R.etl & M.BNC1 & M.lot & R.leg & S.mel & B.jap \\
\hline FegA & $1(35 \%)$ & $1(32 \%)$ & 0 & $2(33$ and35\%) & $2(42$ and34\%) & $2(84$ and $40 \%)$ \\
FhuA & 1 & 2 & 0 & 2 & 4 & 4 \\
FhuB & 5 & 4 & 2 & 5 & 5 & 1 \\
FhuC & 110 & 74 & 114 & 153 & 113 & 87 \\
FhuD & 1 & 1 & 1 & 1 & 0 & 0 \\
\hline
\end{tabular}

The numbers in the table indicate the number of hits obtained in the genomes with the profile-HMMs of FhuA, FhuB, FhuC and FhuD. The numbers in parenthesis indicate the percentage identity of the hit obtained with the FegA sequence using BLASTp; R.etl: Rhizobium etli CFN42 along with plasmids p42a, p42b, p42c, p42d, p42e, p42f; M.BNC1: Mesorhizobium sp. BNC1 along with plasmids pL1, pL2, pL3; M.lot: sorhizobium loti along with plasmids pMLa, pMLb; R.leg: R. leguminosarum biovar viciae 3841 along with plasmids pRL7, pRL8, pRL9, pRL10, pRL11, pRL12; S.mel: Sinorhizobium meliloti 1021 along with plasmids pSymA, pSymB; B.jap: Bradyrhizobium japonicum USDA110 genome.

Ferrichrome constitutes one of the major hydroxamate siderophores in the soil $^{[24]}$. In silico studies were performed to search specifically the ferriferrichrome uptake machinery homologs (FegAB of $B$. japonicum 61A152 and FhuABCD of E. coli) in sequenced genomes of rhizobia. Based on the percent identity of the hits obtained in BLASTp using FegA as the query sequence, FegA homologs of significant identity were detected in S. meliloti and B. japonicum, but not in Rhizobium sp. and Mesorhizobium sp. (Table 4). We failed to detect FegB homologs in any of the rhizobial genomes. Two homologs of FhuA were present in Mesorhizobium sp. BNC1 (GI: 110632699 and GI: 110633793), 2 in $R$. leguminosarum biovar viciae 3841 (GI: pRL120322, GI: pRL100325) and 1 in $R$. etli CFN42 (GI: 86361199). In addition to that enough FhuBCD homologs were present in them to partner the FhuA homologs. However, FhuBCD homologs were detected in Mesorhizobium loti MAFF303099 inspite of the absence of FhuA homologs in its genome.

Four FhuA homologs were detected each in S. meliloti (GI: 15965976, GI: 15966022, GI: 16263416, GI: 15963968 ) and B. japonicum USDA110 (GI: 27379615, GI: 27380031, GI: 27383079, GI: 27379015) genomes. It was also of importance that FhuD homologs were detected in genomes of Rhizobium sp. and Mesorhizobium sp. studied, while they were absent in B. japonicum and S. meliloti. The rhizobial strain under present study, Rhizobium sp. ST1 failed to utilize hydroxamate siderophores, ferrichrome and rhodotorulic acid (produced by many fungi) and desferal (produced by actinomycetes) as iron sources (Table 3) and hence was selected for the introduction of the $f e g A$ gene encoding ferrichrome receptor from $B$. japonicum $61 \mathrm{~A} 152$ and to check the effect of its expression on rhizospheric colonization by the strain.
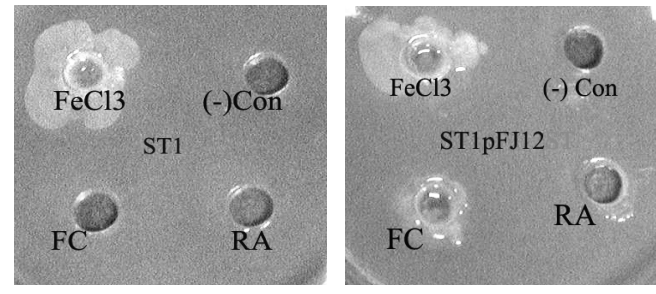

Fig. 1: Ferrichrome (FC) utilization by the transconjugant $\mathrm{ST} 1 \mathrm{pFJ} 12$. The fegA transconjugant ST1pFJ12 exhibits a zone of growth exhibition around the FC $(30 \mu \mathrm{g})$ containing well in contrast to the parent strain Rhizobium sp. ST1

A $2.4 \mathrm{~kb} f e g A$ gene was amplified from B. japonicum 61A152 using fegA gene specific primers (data not shown) and confirmed by sequencing. No $f e g A$ amplification was obtained with genomic DNAs of Rhizobium sp. ST1, Sinorhizobium meliloti IC3169, Mesorhizobium sp. GN25 and Bradyrhizobium sp. NC92 used as templates (data not shown). The B. japonicum 61A152 fegA amplicon was cloned into pUCPM18-Gm vector as per ${ }^{[29]}$ to obtain construct $\mathrm{pFJ}$. fegA (pFJ) was subsequently transformed into E. coli S17.1 (S17.1pFJ) and by patch conjugation with $\mathrm{S} 17.1 \mathrm{pFJ}$ fegA was mobilized into Rhizobium sp. ST1. The transconjugants were screened on Rif-Amp-Gm plates. The presence of $f e g A$ in the transconjugant ST1pFJ12 was confirmed by restriction digestion analysis of the plasmid isolated and by colony PCR of the fegA gene (data not shown). Transconjugant ST1pFJ12 was tested for the cross-utilization of all the siderophores previously tested and as opposed to the parent strain ST1, transconjugant ST1pFJ12 could utilize the siderophore ferrichrome (Fig. 1). A $79 \mathrm{kDa}$ iron regulated outer membrane protein was found to be present in the outer membrane protein preparation of ST1pFJ12 which correlated with the FegA protein of B. japonicum $61 \mathrm{~A} 152^{[30]}$ and was absent in the parent strain ST1 (Fig. 2). This confirmed the expression of FegA in the transconjugant ST1pFJ12. Significant increase in growth of the transconjugant ST1pFJ12 was observed in iron limited conditions, in the presence of pure ferrichrome $(15 \mu \mathrm{M})$ in comparison to its absence (Fig. 3b), while no such growth stimulation due to ferrichrome was seen with parent strain ST1 (Fig. 3a). It was however observed that the presence of ferrichrome partially inhibited the growth of parent ST1 (Fig. 3a). 
OnLine J. Biol. Sci., 9 (2): 40-51, 2009

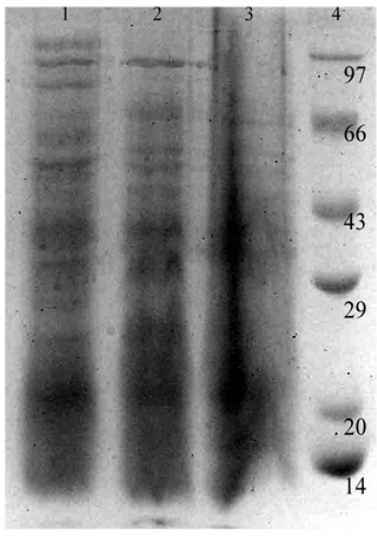

Fig. 2: Outer membrane profiles to check expression of FegA in fegA transconjugant of Rhizobium sp. ST1. (1): B. japonicum 61 A151 Fe (-) condition. (2): transconjugant ST1pFJ12 Fe (-) condition (3): parent strain ST1 Fe(-) condition

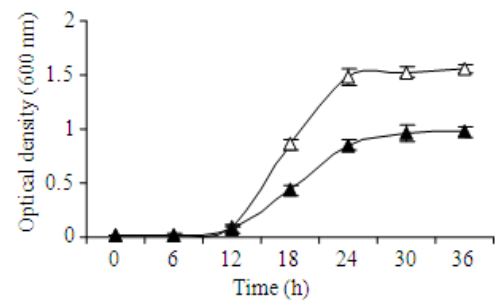

(a)

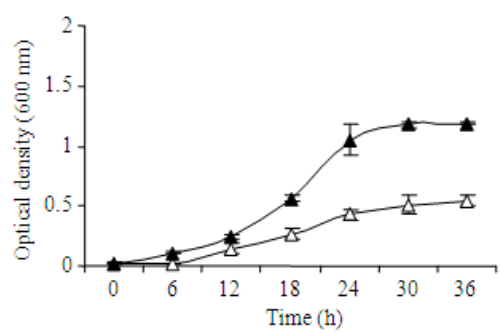

(b)

Fig. 3: Growth of (a): ST1 and (b): fegA transconjugant $\mathrm{ST} 1 \mathrm{pFJ} 12$ in the presence (filled triangles) and absence (open triangles) of externally supplemented ferrichrome $(15 \mu \mathrm{M})$. The values represent mean of three independent experiments, vertical bars indicate standard deviation

Pot inoculation studies were performed under natural soil conditions in order to check the potential of the transconjugant ST1pFJ12 for rhizospheric colonization and nodulation on pigeon pea, the nodulating host of ST1. Pigeon pea seedlings inoculated with $\mathrm{ST} 1 \mathrm{pFJ} 12$ produced significant increase in shoot

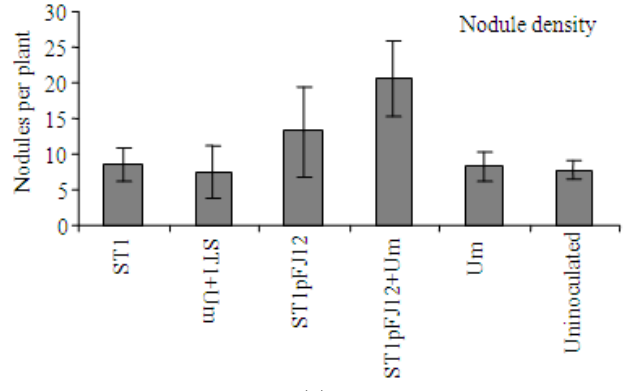

(a)

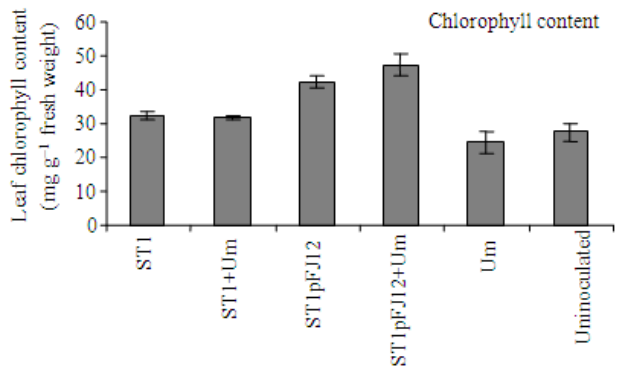

(b)

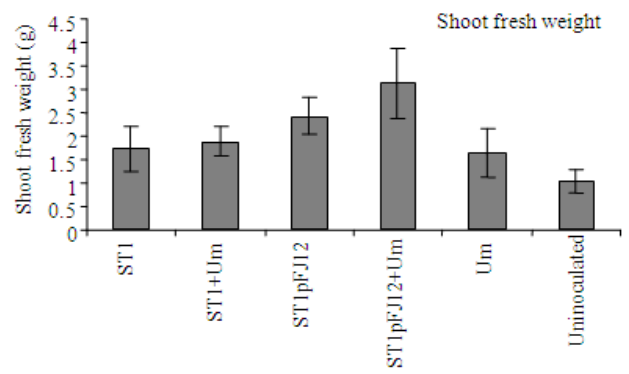

(c)

Fig. 4: Influence of $f e g A$ transconjugant $\mathrm{ST} 1 \mathrm{pFJ} 12$ inoculation on pigeon pea plant parameters as compared to parent strain Rhizobium sp. ST1 inoculation in natural soil condition, in the presence and absence of ferrichrome producing fungus Ustilago maydis. Plants were harvested 30 days post inoculation and were assayed for average chlorophyll content of leaves, average shoot fresh weight and average nodule density

and root fresh weight, nodule density $(\mathrm{p}<0.001)$ and chlorophyll content $(\mathrm{p}<0.05)$ of the leaves as compared to ST1 inoculation or uninoculated control plants, the difference being more prominent when $U$. maydis was co-inoculated in the systems (Fig. 4). This increase in plant parameters correlated with the counts of the inoculated bacteria in the rhizospheric soil which were estimated at start of the experiment and at the end of 30 days post-inoculation on selective antibiotic plates. 
Table 5: Nodule occupancy by Rhizobium sp. ST1 and transconjugant ST1pFJ12 in the presence and absence of Ustilago maydis inoculated under natural soil conditions

\begin{tabular}{lclll}
\hline Condition & $\begin{array}{l}\text { Total No. } \\
\text { of nodules }\end{array}$ & $\begin{array}{l}\text { No. of nodule } \\
\text { isolates growing } \\
\text { on antibiotic plates }\end{array}$ & $\begin{array}{l}\text { Effective } \\
\text { nodulation by } \\
\text { inoculated strains }\end{array}$ & $\begin{array}{l}\text { Nodule } \\
\text { occupancy } \\
(\%)\end{array}$ \\
\hline ST1 & 69 & $31($ Rif) & 26 & 37 \\
ST1+Um & 71 & $33(\mathrm{Rif})$ & 28 & 30 \\
ST1pFJ12 & 84 & $58(\mathrm{Rif} / \mathrm{Amp} / \mathrm{Gm})$ & 51 & 57 \\
ST1pFJ12+Um & 112 & $82(\mathrm{Rif} / \mathrm{Amp} / \mathrm{Gm})$ & 75 & 66 \\
Um & 49 & 6 (Rif); 6 (Amp/Gm) & \\
Uninoculated & 46 & $4(\mathrm{Rif}) ; 8(\mathrm{Amp} / \mathrm{Gm})$ & \\
\hline
\end{tabular}

Starting with approximately $8.6 \times 10^{4} \quad \mathrm{cfu} \quad \mathrm{g}^{-1}$ rhizospheric soil, the ST1 population decreased to $2.1 \times 10^{3}$ and $1.9 \times 10^{2} \mathrm{cfu} \mathrm{g}^{-1}$ rhizospheric soil in the absence and presence of $U$. maydis, respectively. In contrast to this ST1pFJ12 was able to increase their populations to $1.0 \times 10^{5}$ and $3.4 \times 10^{5}$, when inoculated alone and when co-inoculated with $U$. maydis, respectively, starting with an initial population of $7.9 \times 10^{4}$. The nodule occupancy, as calculated by scoring the pure cultures isolated from nodules on antibiotic marker plates, was found to be $57 \%$ and $37 \%$ on the plants inoculated with transconjugant ST1pFJ12 and parent strain ST1, respectively (Table 5 ). No significant difference in the fresh weight of pigeon pea plants was observed when $U$. maydis was co-inoculated with Rhizobium sp. ST1 ( $\mathrm{p}=0.924)$, but a slight decrease in nodule occupancy was observed (Table 5). However $U$. maydis when co-inoculated with transconjugant ST1pFJ12, showed increase in the nodule occupancy to $66 \%$, with no significant effect on shoot fresh weight but an increase in the chlorophyll contents of the leaves. Thus a positive correlation between nodule occupancy and chlorophyll content of the leaves of pigeon pea was observed.

\section{DISCUSSION}

The plant rhizosphere is a dynamic environment where intense competitive interactions take place among microorganisms and many factors affect the microbial composition, the main among these being iron. The iron acquisition problem is more pronounced in rhizobia because nitrogen fixation involves iron rich proteins like nitrogenase, leghemoglobin and cytochromes, with nitrogenase and leghemoglobin constituting up to 12 and $30 \%$ of total protein in the bacterial and infected plant cells, respectively ${ }^{[43]}$. Earlier studies from our lab have demonstrated that Arachis hypogea and Cajanus cajan root nodule isolates of rhizobia are poor producers and utilizers of hydroxamate-type siderophores ${ }^{[26-29,47]}$. Recent study from our laboratory has shown that the expression of Bradyrhizobium japonicum 61A152 fegA gene in peanut rhizobia, not only supports the growth of the fegA transconjugants under iron limited laboratory conditions, but also increases its survivability under natural soil conditions, leading to higher nodulation on peanut plant ${ }^{[31]}$. Similar study presented here regarding the expression of $B$. japonicum fegA in pigeon pea rhizobia, Rhizobium sp. ST1, not only substantiates the earlier results, but also allows us to hypothesize that hydroxamate siderophore utilization can be considered as an important criteria while selecting a bioinoculant strain. In order to determine the relative importance of ferrichrome utilization, we have also carried out an in silico investigation of the occurrence of ferrichrome receptor proteins in sequenced genomes of various rhizobia.

Rhizobial strains investigated here were found to produce and utilize catechol-type siderophores, but were poor producers and utilizers of hydroxamate-type siderophores. In contrast to our observations, majority of the rhizobial inoculant strains for Australian pulse and legume crops from the Australian Inoculants Research and Control Service (AIRCS) are reported to be producers of either tri- or di-hydroxamate siderophores and are successfully used as commercial inoculants for pulse and legume pastures ${ }^{[45]}$. Field tests performed with B. japonicum 61A152, consistently gave high yields of soybean cultivars ${ }^{[22]}$ and this led to the speculation that iron-scavenging property of B. japonicum 61A152 may be responsible for its competitive survival in the rhizosphere and hence better nodulation.

The $f e g A$ gene with its native promoter was cloned into pUCPM18-Gm (pFJ) and when transferred into Mesorhizobium sp. GN25 ${ }^{[29]}$ and Rhizobium sp. ST1 (present study), imparted the ability to utilize ferrichrome, implying that the ferrichrome transport through the outer membrane could be complemented by the inner membrane protein machinery already present in these organisms. A finding similar to ours, where expression of only the outer membrane receptor imparts siderophore utilization has been reported where the expression of fauA gene, encoding receptor for alcaligin siderophore, imparts alcaligin utilization to a $P$. aeruginosa strain deficient in alcaligin production $^{[46]}$ and introduction of fhuA gene brings about ferrichrome utilization in rhizobial strains deficient of ferrichrome production as well as utilization $^{[47]}$. Growth stimulation of only the transconjugant ST1pFJ12 and not the parent strain ST1 in the presence of externally added ferrichrome, under iron limited growth conditions implies that, as opposed to the parent strain ST1, the transconjugant has a clear growth advantage. Hydroxamate type siderophores are 
the main amongst the siderophores found in soil ${ }^{[23]}$ most of it being of ferrichrome type, present in nanomolar concentrations ${ }^{[24]}$. Rhizobium sp. ST1 would therefore be at a competitive disadvantage when in direct competition with other rhizospheric organisms, most of them reported to be able to utilize iron bound to hydroxamates ${ }^{[25]}$. Present results provide evidence that engineering rhizobial strains with ferrichrome utilization ability add a competitive edge in an environment where ferri-ferrichrome is the main available source of iron.

It was surprising that we detected FhuA homologs in genomes of S. meliloti, Mesorhizobium sp. BNCI and $R$. etli but the laboratory isolates of the corresponding genera failed to utilize ferrichrome. Possession of genetic elements for ferrichrome utilization system even differed among the two strains of mesorhizobia analyzed in this study. Rhizobiales are reported to have large genomes with disproportionate enrichment of high-affinity nutrient acquisition and secondary metabolism $^{[50,51]}$. This genome plasticity is predicted to be of evolutionary advantage in soil environments where resources are scarce but diverse ${ }^{[51,52]}$ and intraspecific genetic variation contributes to niche adaptation. Our results of inconsistency of ferrichrome cross-utilization, among members of the same genus, were justifiable in light of the above reports. In Mesorhizobium sp. BNC1, out of the 2 FhuA homologs identified by profile-HMM search, one was a TonBdependent heme/hemoglobin receptor family protein (GI: 110633793) and FhuBCD homologs (GI: 110633795; GI: 110633796; GI: 110633797) were also found in close proximity, most probably organized to form an operon. The other FhuA homolog (GI: 110633699), encoded a TonB dependent siderophore receptor, was not associated with any of the FhuBCD homologs detected. R. leguminosarum biovar Viciae among the rhizobia, has been shown to have a Fhu PBT (Periplasmic Binding protein-dependent Transport) system for the uptake of trihydroxamate siderophore vicibactin $^{[5]}$. In our analysis of $R$. leguminosarum genome, the iron-siderophore uptake machinery was present on plasmid pRL, different from other rhizobia, where the iron uptake machinery was present on the genome and as already reported ${ }^{[5]}$ were clustered together as FhuABDC (GI: pRL100325, GI: pRL100326, GI: pRL100327 and GI: pRL100328). One FhuD homologue each was also present in genomes of Rhizobium sp. and Mesorhizobium sp. and a closer look at the genome sequence suggested the presence of FhuPBT type siderophore uptake system, associated with at least one FhuA homolog in Rhizobium and Mesorhizobium sp. The other FhuA homologs were not found to be associated with inner membrane machinery, similar to FhuE (rhodotorulic acid and coprogen receptor) and IutA (aerobactin receptor) of E. coli, which are also found in isolation ${ }^{[4]}$. These receptors work in association with FhuBCD (ferrichrome system) suggest that the transport of ferri-siderophores through the inner membrane is not as specific as that through the outer membrane. It is due to this reason that a notably less number of periplasmic and cytoplasmic membrane proteins are reported to be present in Bradyrhizobium sp. and Pseudomonas sp. against a relatively large number of outer membrane receptors reported $^{[2]}$. Four homologs of FhuA in B. japonicum USDA110 (GI: 27379615; GI: 27380031; GI: 27383079; GI: 27379015) and one in S. melitoti (GI: 16263416) were detected. RhtX and FptX are members of a novel family of permeases for the transport of rhizobactin and ferricpyochelin respectively, through the inner membrane ${ }^{[3]}$. Though FegB does not share any sequence similarity to RhtX or FptX, it may be acting in a similar manner to transport ferrichrome across the inner membrane ${ }^{[2]}$. We failed to find homologs of FhuD in $B$. japonicum and $S$. meliloti in support to the above observations that transport of ferrisiderophores through the inner membrane in S. meliloti and B. japonicum is FhuD independent, dissimilar to the Fhu-PBT uptake system of E. coli. Because FhuC is a cytoplasmic ATPase and ATPases are highly conserved proteins, they were detected in very high numbers as compared to permease proteins which had unique sequences that made them specific for classes of siderophores or various other molecules which are also transported through $\mathrm{ABC}$ transporter permeases.

The successful performance of rhizobial inoculant strains depends upon their capability to out compete the indigenous soil bacteria, survive, propagate and enter into effective symbiosis with the host plant. Biofertilizer strains which fail to survive under soil conditions are most of the times ineffective in enhancing legume productivity because vast majority of nodules formed are not by the inoculated strain, but by indigenous rhizobia in the soil ${ }^{[6,7,44]}$. Rhizobium sp. ST1 was inhibited slightly in the presence of ferrichrome under lab conditions and also under natural soil conditions, which also gets reflected on its nodule occupancy on pigeon pea plants when co-inoculated with $U$. maydis. This might be because ST1 is unable to utilize ferrichrome and it could be argued here that the siderophore it itself produces is of a significantly lower affinity then ferrichrome ${ }^{[26]}$. Our results of plant inoculation state that expression of $f e g A$ into Rhizobium sp. ST1 increases the rhizospheric stability of the resultant transconjugant, which is evident by increase in 
nodule occupancy on pigeon pea plants. It is demonstrated that having many nodules occupied by the inoculated rhizobial strains (ST1pFJ12) have positive effects on growth, nodulation and chlorophyll content of the plants and hence it is more likely that it would even affect legume yield. Previous reports have shown that co-inoculation of $B$. japonicum USDA110 with pseudomonads producing siderophores utilizable by the strain, enhances its nodulation frequency even in the presence of other competing bradyrhizobial strains ${ }^{[48]}$. Several rhizobial species have been reported to produce siderophores under culture conditions ${ }^{[10,11]}$, but there is no evidence that these siderophores are made in planta. There are also evidences that mature $\mathrm{N}_{2}$-fixing bacteroids of $R$. leguminosarum do not transcribe the vicibactin uptake receptor gene at detectable levels $^{[49]}$. It is possible that FegA also does not have anything to do with iron uptake in planta ${ }^{[2]}$. Whether siderophore production or uptake efficiency increases the nitrogen fixation ability of nodule bacteria is not yet known, but their survival in the absence of their host definitely depends upon this characteristic.

\section{CONCLUSION}

Because ferrichrome is synthesized by a variety of soil fungi, it is likely to be the main iron source in the rhizosphere. Expression of fegA in Rhizobium sp. ST1, not only imparted upon the strain ability to utilize ferrichrome, but also increased its survivability in the soil, substantiating the hypothesis put forward. Coinoculation of ferrichrome producing $U$. maydis did not have any antagonistic effect on plant growth, however transconjugant ST1pFJ12 co-inoculated with $U$. maydis do show better survival and hence increased nodule occupancy, as compared to parent ST1, co-inoculated with $U$. maydis. The results obtained here in association with other similar reports from our laboratory ${ }^{[26-29,47]}$ support the fact that iron sufficiency of any organism largely depends on its ability to utilize the siderophore present in large concentrations in its vicinity ${ }^{[2,48]}$. Survival under iron limiting soil conditions is an important quality which every biofertilizer strain must possess. We therefore conclude that iron availability is one of the major factors determining rhizospheric colonization and therefore in order to select an efficient biofertilizer strain, its hydroxamate siderophore utilization property also holds a prime importance.

\section{ACKNOWLEDGEMENT}

Hearty thanks to Mary. L Guerinot for providing Bradyrhizobium japonicum 61A152 strain. Council of
Scientific and Industrial Research, Government of India is acknowledged for the fellowship provided to FRJ. Part of this study was supported by University Grants Commission (UGC sanction no: F.No.3-49/2001 (SR-I) dated 31-3-04) and also by Department of Biotechnology (DBT) New Delhi, grant to A.J.D. and G.A. (no: BT/PR2420/AGR/21/118/2001).

\section{REFERENCES}

1. Guerinot, M.L., 1991. Iron uptake and metabolism in the rhizobia/legume symbioses. Plant. Soil, 130: 199-209. DOI: 10.1007/BF00011874

2. Benson, H.P., E. Boncompagni and M.L. Guerinot, 2005. An iron uptake operon required for proper nodule development in the Bradyrhizobium japonicum-soybean symbiosis. Mol. Plant Microbe Ineract., 18: 950-959. DOI: 10.1094/MPMI-180950

3. Cuív, P.Ó., P. Clarke, D. Lynch and M. O’Connell, 2004. Identification of $r h t X$ and $f p t X$, novel genes encoding proteins that show homology and function in the utilization of the siderophores rhizobactin 1021 by Sinorhizobium meliloti and pyochelin by Pseudomonas aeruginosa, respectively. J. Bacteriol., 186: 2996-3005. DOI: 10.1128/JB.186.10.2996-3005.2004

4. Braun, V., 1995. Energy-coupled transport and signal transduction through the gram-negative outer membrane via TonB-ExbB-ExbD-dependent receptor proteins. FEMS. Microbiol. Rev., 16: 295-307. DOI: 10.1016/0168-6445(95)00003-U http://www.ncbi.nlm.nih.gov/pubmed/3301821

5. Stevens, J.B., R.A. Carter, H. Hussain, K.C. Carson, M.J. Dilworth and A.W. B. Johnston, 1999. The fhu genes of Rhizobium leguminosarum, specifying siderophore uptake proteins: fhuDCB are adjacent to a pseudogene version of fhuA. Microbiology, 145: 593-601.

http://cat.inist.fr/?aModele $=$ afficheN\&cpsidt $=1779$ 677

6. Hemantaranjan, A. and O.K. Garg, 1986. Introduction of nitrogen fixing nodules through iron and zinc fertilization in the non noduleforming French bean (Phaseolus vulgaris L.). J. Plant Nutr., 9: 281-288. http://cat.inist.fr/?aModele $=$ afficheN\&cpsidt $=8051771$

7. Streeter, J.G., 1994. Failure of inoculant rhizobia to overcome the dominance of indigenous strains for nodule formation. Can. J. Microbiol., 40: 513-522. http://cat.inist.fr/?aModele $=$ afficheN\&cpsidt $=4162$ 423 
8. Smith, M.J., J.N. Schoolery, B. Schwyn, I. Holden and J.B. Neilands, 1985. Rhizobactin, a structurally novel siderophore from Rhizobium meliloti. J. Am. Chem. Soc., 107: 1739-1743. DOI: 10.1021/ja00292a047

9. Lynch, D., J. O'Brien, T. Welch, P. Clarke, P.O. Cuiv, J.H. Crosa and M. O'Connell, 2001. Genetic organization of the region encoding regulation, biosynthesis and transport of rhizobactin 1021, a siderophore produced by Sinorhizobium meliloti. J. Bacteriol., $\quad$ 183: 2576-2585. DOI: 10.1128/JB.183.8.2576-2585.2001

10. Persmark, M., P. Pittman, J.S. Buyer, B. Schwyn, R. Gill and J.B. Neilands, 1993. Isolation and structure of rhizobactin 1021, a siderophore from the alfalfa symbiont Rhizobium meliloti 1021. J. Am. Chem. Soc., 115: 3950-3956. DOI: 10.1021/ja00063a014

11. Dilworth, M.J., K.C. Carson, R.G.F. Giles, L.T. Byrne and A.R. Glenn, 1998. Rhizobium leguminosarum bv. viciae produces a novel cyclic trihydroxamate siderophore, vicibactin. Microbiology, 144: 781-791. http://cat.inist.fr/?aModele=afficheN\&cpsidt=2221298

12. Berraho, E.L., D. Lesueur, H.G. Diem and A. Sasson, 1997. Iron requirement and siderophore production in Rhizobium ciceri during growth on an irondeficient medium. World J. Microbiol. Biotech., 13: 501-510. DOI: 10.1023/A:1018553022960

13. Jadhav, R.S. and A.J. Desai, 1992. Isolation and characterization of siderophore from cowpea Rhizobium (peanut isolate). Curr. Microbiol., 24: 137-141. DOI: 10.1007/BF01568978

14. Modi, M., K.S. Shah and V.V. Modi, 1985. Isolation and characterization of catechol like siderophores from cowpea Rhizobium RA-1. Arch. Microbiol., 141 : 156-158. DOI: 10.1007/BF00423277

15. Manjanatha, M.G., T.E. Loynachan and A.G. Atherly, 1992. Tn5 mutagenesis of Chinese Rhizobium fredii for siderophore overproduction. Soil Biol. Biochem., $24:$ 151-155. http://cat.inist.fr/?aModele $=$ afficheN\&cpsidt $=5205160$

16. Barran, L.R. and E.S.P. Bromfield, 1993. Does siderophore production influence the relative abundance of Rhizobium meliloti in two field populations? Can. J. Microbiol., 39: 348-351. DOI: 10.1139/CJM-39-3-348

17. Dean, C.R. and K. Poole, 1993. Cloning and characterization of the ferric enterobactin receptor gene (pfeA) of Pseudomonas aeruginosa. J. Bacteriol., 175: 317-324. http://www.pubmedcentral.nih.gov/articlerender.fc gi?artid=196144
18. Ankenbauer, R.G. and H.N. Quan, 1994. FptA, the $\mathrm{Fe}(\mathrm{III})$-pyochelin receptor of Pseudomonas aeruginosa: A phenolate siderophore receptor homologous to hydroxamate siderophore receptors. J. Bacteriol., 176: 307-319. http://jb.asm.org/cgi/reprint/176/2/307

19. Cornelis, P. and S. Matthijs, 2002. Diversity of siderophore mediated iron uptake systems in fluorescent pseudomonads: not only pyoverdines. Environ. Microbiol., 4: 787-798. DOI: 10.1046/j.1462-2920.2002.00369.x

20. Guerinot, M.L., E.J. Meidl and O. Plessner, 1990. Citrate as a siderophore in Bradyrhizobium japonicum. J. Bacteriol., $\quad$ 172: 3298-3303. http://www.pubmedcentral.nih.gov/articlerender.fc gi? artid=209139

21. Plessner, O., T. Klapatch and M.L. Guerinot, 1993. Siderophore utilization by Bradyrhizobium japonicum. Applied Environ. Microbiol., 59: 1688-1690. http://www.pubmedcentral.nih.gov/articlerender.fc gi? artid $=182140$

22. Hume, D.J. and B.J. Shelp, 1990. Superior performance of the Hup- Bradyrhizobium japonicum strains $532 \mathrm{C}$ in Ontario soybean field trials. Can. J. Plant Sci., 70: 661-666.

23. Powell, P.E., G.R. Cline, C.P.P. Reid and P.J. Szaniszlo, 1980. Occurance of hydroxamate siderophore iron chelators in soils. Nature, 287: 833-834. DOI: 10.1038/287833a0

24. Powell, P.E., P.J. Szaniszlo and C.P.P. Reid, 1983. Confirmation of occurance of hydroxamate siderophores in soil by a novel Escherichia coli bioassay. Appl. Environ. Microbiol., 46: 1080-1083. http://www.pubmedcentral.nih.gov/articlerender.fcgi? artid $=239522$

25. Jurkevitch, E., Y. Hadar and Y. Chen, 1992. Differential siderophore utilization and iron uptake by soil and rhizosphere bacteria. Applied Environ. Microbiol., 58: 119-124. http://www.pubmedcentral.nih.gov/articlerender.fc gi?artid=195181

26. Joshi, F.R., G. Archana and A.J. Desai, 2006. Siderophore cross-utilization amongst rhizospheric bacteria and role of their differential affinities for $\mathrm{Fe}^{3+}$ on growth stimulation under iron limited conditions. Curr. Microbiol., 53: 141-147. http://www.ncbi.nlm.nih.gov/pubmed/16845564

27. Khan, A., R. Geetha, A. Akolkar, A. Pandya, G. Archana and A.J. Desai, 2006. Differential cross-utilization of heterologous siderophores by nodule bacteria of Cajanus cajan and its, possible role in growth under iron-limited conditions. Applied Soil. Ecol., 34: 19-26. DOI: 10.1016/j.apsoil.2005.12.001 
28. Joshi, F., S. Kholiya, G. Archana and A.J. Desai, 2008. Siderophore cross-utilization amongst nodule isolates of the cowpea miscellany group and its effect on plant growth in the presence of antagonistic organisms. Microbiol. Res., 163: 564-570. DOI: 10.1016/j.micres.2006.08.004

29. Joshi, F.R., A. Chaudhari, P. Joglekar, G. Archana and A.J. Desai, 2008b. Effect of expression of Bradyrhizobium japonicum 61A152 fegA gene in Mesorhizobium sp., on its competitive survival and nodule occupancy on Arachis hypogea. Appl. Soil. Eco., 40: 338-347. DOI:10.1016/j.apsoil.2008.06.003

30. Levier, K. and M.L. Guerinot, 1996. The Bradyrhizobium japonicum fegA gene encodes an iron regulated outer membrane protein with similarity to hydroxamate-type siderophore receptors. J. Bacteriol., 178: 7265-7275. http://jb.asm.org/cgi/reprintframed/178/24/7265

31. Winklemann, G., 1986. Iron Complex Compounds (Siderophores). In: Biotechnology Microbial Products II, Pape, H. and H.J. Rehm (Eds.). VCH Publishers, New York, pp: 215-243.

32. Arnow, L.E., 1937. Colorimetric determination of the components of 3,4-dihydroxy phenylalanine tyrosine mixtures. J. Biol. Chem., 118: 531-535. http://www.jbc.org/cgi/reprint/118/2/531.pdf

33. Gibson, F. and D.I. Magrath, 1969. The isolation and characterization of hydroxamic acid (aerobactin) formed by Aerobacter aerogenes 62-I. Biochim. Biophys. Acta, 192: 175-184. http://www.ncbi.nlm.nih.gov/pubmed/4313071

34. Apweiler, R., A. Bairoch, C.H. Wu, W.C. Barker and B. Boeckmann et al., 2004. UniProt: The Universal Protein Knowledgebase. Nucl. Acids Res., 32: 115-119. http://nar.oxfordjournals.org/cgi/content/full/32/sup pl_1/D115

35. Edgar, R.C., 2004. MUSCLE: multiple sequence alignment with high accuracy and high throughput. Nucl. Acid. Res., 32: 1792-1797. http://www.ncbi.nlm.nih.gov/pubmed/15034147

36. Altschul, S.F., W. Gish, W. Miller, E.W. Myers and D.J. Lipman, 1990. Basic local alignment search tool. J. Mol. Biol., 215: 403-410. http://www.ncbi.nlm.nih.gov/pubmed/2231712

37. Hester, K.L., L. Jodi, F. Najar, L. Song and B.A. Roe et al., 2000. Crc is involved in catabolite repression control of the bkd operons of Pseudomonas putida and Pseudomonas aeruginosa. J. Bacteriol., 182: 1144-1149. http://jb.asm.org/cgi/content/abstract/182/4/1144
38. Sambrook, J. and D.W. Russell, 2001. Molecular Cloning. A Laboratory Manual. 3rd Edn., Cold Spring Harbor Laboratory Press, Cold Spring Harbor, New York, ISBN: 0879695773, pp: 2100.

39. Simon, R., 1984. High frequency mobilization of gram-negative bacterial replicon by the in vitro constructed Tn5 mob transposon. Mol. Gen. Genet., 196: 413-420. http://www.ncbi.nlm.nih.gov/pubmed/6094969

40. Guan, L.L., K. Kanoh and K. Kamino, 2001. Effect of exogenous siderophores on iron uptake activity of marine bacteria under iron-limited conditions. Applied Environ. Microbiol., 67: 1710-1717. DOI: 10.1128/AEM.67.4.1710-1717.2001

41. Schwyn, B. and J.B. Neilands, 1987. Universal chemical assay for the detection and determination of siderophores. Anal. Biochem., 160: 47-56. http://www.ncbi.nlm.nih.gov/pubmed/2952030

42. Woestyne, M.V., B. Bruyneel, M. Mergeay and W. Verstraete, 1991. The $\mathrm{Fe}^{+2}$ chelator proferrorosamine $A$ is essential for the siderophore mediated uptake of iron by Pseudomonas roseus fluorescens. Applied Environ. Microbiol., 57: 949-954. http://www.pubmedcentral.nih.gov/articlerender.fc gi? $\operatorname{artid}=182828$

43. Verma, D.P.S. and S. Long, 1983. The molecular biology of Rhizobium-legume symbiosis. Int. Rev. Cytol. $\quad$ Suppl., $\quad$ 14: 211-245. http://www.fao.org/agris/search/display.do?f=./198 4/v1003/US8273791.xml;US8273791

44. O'Hara, G.W., M.J. Dilworth, N. Boonkerd and P. Parkpian, 1988. Iron deficiency specifically limits nodule development in peanut inoculated with Bradyrhizobium sp. New Phytol., 108: 51-57. DOI: 10.1111/j.1469-8137.1988.tb00203.x

45. Carson, K.C., J.M. Meyer and M.J. Dilworth, 2000. Hydroxamate siderophores of root nodule bacteria. Soil. Biol. Biochem., 32: 11-21. DOI: 10.1016/S0038-0717(99)00107-8

46. Brickman, T.J. and S.K. Armstrong, 1999. Essential role of the iron-regulated outer membrane receptor fauA in alcaligin siderophore-mediated iron uptake in Bordetella sp. J. Bacteriol., 181: 5958-5966.

http://cat.inist.fr/?aModele $=$ afficheN\&cpsidt $=1025$ 5709

47. Rajendran, G., S. Mistry, A.J. Desai and G. Archana, 2007. Functional expression of Escherichia coli fhuA gene in Rhizobium spp. of Cajanus cajan provides growth advantage in presence of $\mathrm{Fe}+3$ : ferrichrome as iron source. Arch. Microbiol., 187: 257-264. DOI: $10.1007 / \mathrm{s} 00203-006-0191-8$ 
48. Fuhrmann, J. and A.G. Wollum, 1989. Nodulation competition among Bradyrhizobium japonicum strains as influenced by rhizosphere bacteria and iron availability. Biol. Fertility Soil, 7: 108-112. DOI: $10.1007 / \mathrm{BF} 00292567$

49. Yeoman, K., F. Wisniewski-Dye, C. Timony, J. Stevens, N. deLuca, J.A. Downie and A.W.B. Johnston, 2000. Analysis of the Rhizobium leguminosarum siderophore-uptake gene fhuA: Differential expression in free-living bacteria and nitrogenfixing bacteroids and distribution of $f h u A$ pseudogene in different strains. Microbiology, 146: 829-837.

http://mic.sgmjournals.org/cgi/content/abstract/146 /4/829

50. Boussau, B., E.O. Karlberg, A.C. Frank, B.A. Legault and S.G. Andersson, 2004. Computational inference of scenarios for alpha-proteobacterial genome evolution. Proc. Natl. Acad. Sci. USA., 101: 9722-9727. DOI: 10.1073/pnas.0400975101
51. Konstantinidis, K.T. and J.M. Tiedje, 2004. Trends between gene content and genome size in prokaryotic species with larger genomes. Proc. Natl. Acad. Sci. USA., 101: 3160-3165. DOI: 10.1073/pnas.0308653100

52. Gonzalez, V., R.I. Santamaria, P. Bustos, I. Hernandez-Gonzalez, A. Medrano-Soto and G. Moreno-Hagelsieb et al., 2006. The partitioned Rhizobium etli genome: genetic and metabolic redundancy in seven interacting replicons. Proc. Natl. Acad. Sci. USA., 103: 3834-3839. DOI: 10.1073/pnas.0508502103 